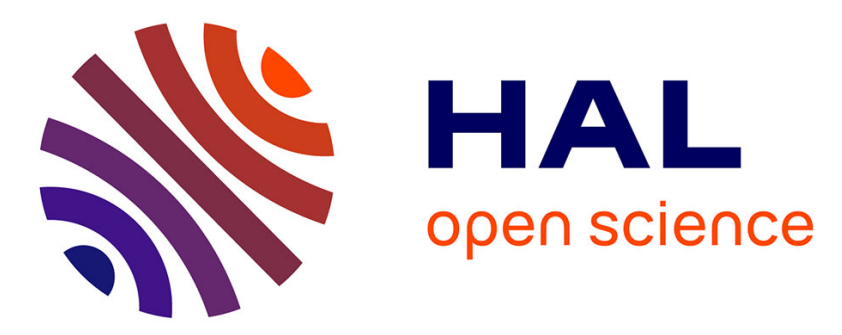

\title{
Utilisation d'ions lithium pour l'analyse des surfaces et des couches minces
}

\author{
J.-P. Thomas, A. Cachard, M. Fallavier, J. Tardy, S. Marsaud, J. Pivot
}

\section{To cite this version:}

J.-P. Thomas, A. Cachard, M. Fallavier, J. Tardy, S. Marsaud, et al.. Utilisation d'ions lithium pour l'analyse des surfaces et des couches minces. Revue de Physique Appliquée, 1976, 11 (1), pp.65-72. 10.1051/rphysap:0197600110106500 . jpa-00244044

\section{HAL Id: jpa-00244044 https://hal.science/jpa-00244044}

Submitted on 1 Jan 1976

HAL is a multi-disciplinary open access archive for the deposit and dissemination of scientific research documents, whether they are published or not. The documents may come from teaching and research institutions in France or abroad, or from public or private research centers.
L'archive ouverte pluridisciplinaire HAL, est destinée au dépôt et à la diffusion de documents scientifiques de niveau recherche, publiés ou non, émanant des établissements d'enseignement et de recherche français ou étrangers, des laboratoires publics ou privés. 


\title{
UTILISATION D'IONS LITHIUM POUR L'ANALYSE DES SURFACES ET DES COUCHES MINCES (*)
}

\author{
J. P. THOMAS $(* *)$, A. CACHARD $(* * *)$, M. FAllaVieR $(* *)$, J. TARDY $(* * *)$, \\ S. MARSAUD $(* *)$ et J. PIVOT $(* * *)$ \\ Université Claude-Bernard Lyon-I, 43, bd du 11-Novembre-1918, 69621 Villeurbanne, France
}

\begin{abstract}
Résumé. - Dans la tendance actuelle à utiliser des ions plus lourds que les particules $\alpha$ en rétrodiffusion élastique (domaine d'énergie de l'ordre du MeV), l'emploi d'ions lithium apparaît constituer une amélioration notable des caractéristiques essentielles de la méthode. Ceci est particulièrement vrai en ce qui concerne la résolution en profondeur, avec le maintien de la résolution des détecteurs, pour ce type d'ions. Nous présentons ici, en même temps qu'une étude comparative des performances analytiques des ions $\mathrm{Li}$ et d'autres particules, quelques applications en montrant l'intérêt dans l'étude des surfaces et des couches minces. Parmi les applications les plus caractéristiques, citons, pour des échantillons épais, l'étude simultanée de la composition des couches d'oxydation anodique formées à la surface d'aluminium et du comportement de l'impureté majeure du bain de formation. Dans les études de couches minces, outre la détermination précise de la stœchiométrie des couches diélectriques $\mathrm{AlN}, \mathrm{SiO}, \mathrm{Al}_{2} \mathrm{O}_{3}$, des effets d'implantation pourront être étudiés, comme l'oxygène dans les couches $\mathrm{SiO}$.
\end{abstract}

\begin{abstract}
In the actual trend to use ions heavier than $\alpha$-particles in backscattering (MeV energy range) lithium ions appear as to lead to a noticeable improvement of the main characteristics of the method. This is especially pointed out with the depth resolution owing to the fact that detector resolution is maintained for this type of particle. We present here, together with a comparative study of the analytical performances of $\mathrm{Li}$ ions and other particles, some applications devoted to surface and thin film studies. Among the most characteristic applications, for thick samples, anodic oxidation layers of aluminium can be characterized through the aluminium and the solution main impurity distributions. For thin film studies, precise stoichiometry determination can be achieved for the dielectric layers $\mathrm{AlN}, \mathrm{SiO}, \mathrm{Al}_{2} \mathrm{O}_{3}$ and implantation effects can be followed like for oxygen in $\mathrm{SiO}$.
\end{abstract}

1. Introduction. - Parmi les méthodes de microanalyse appliquées aux études de surface et de couches minces, la rétrodiffusion élastique apparaît comme très largement utilisée, compte tenu de la relative simplicité de la mise en cuvre et de l'interprétation des spectres obtenus. L'emploi des protons [1] mais surtout des particules alpha, est très répandu, comme en témoigne le nombre important de travaux publiés au cours des cinq dernières années [2]. Le recours à des particules plus lourdes peut cependant apporter une amélioration notable aux caractéristiques essentielles de la méthode : sélectivité, sensibilité et résolution en profondeur. Les ions ${ }^{12} \mathrm{C},{ }^{14} \mathrm{~N},{ }^{16} \mathrm{O}$ ont ainsi été utilisés par divers auteurs [3], [4] mais ont fait apparaître une restriction importante au niveau de la résolution des détecteurs (barrière de surface) classiquement utilisés. L'emploi d'ions lithium 6 ou 7 non seulement offre un bon compromis en sensibilité entre particules alpha et ions plus lourds, mais permet en outre un gain de sélectivité appréciable pour les éléments de $\mathbf{Z}$ moyen. De plus, et c'est un des intérêts majeurs de ce

$\left(^{*}\right)$ Travaux effectués avec le soutien de la D. G. R. S. T. (contrat 73-7-1545) et du C. N. R. S. (ATP No 1 E 11).

$\left({ }^{* *}\right)$ Institut de Physique Nucléaire.

(***) Département de Physique des Matériaux. type de particule, l'excellente résolution maintenue dans le détecteur autorise des résolutions en profondeur parmi les plus performantes de la méthode. Ces différents aspects sont illustrés ici dans l'étude de couches d'oxydation anodique formées à la surface d'échantillons massifs d'aluminium et dans l'analyse de couches minces diélectriques d'oxyde et nitrure d'aluminium ainsi que d'oxyde de silicium. Avec, dans certains cas, une confrontation des résultats obtenus à partir d'autres méthodes, une discussion est faite des limitations et des améliorations qu'on peut apporter à une telle utilisation d'ions lourds légers.

2. Caractéristiques de la rétrodiffusion élastique d'ions lithium. - Les trois caractéristiques déjà mentionnées dépendent évidemment de l'énergie, mais aussi de la nature de l'ion incident. La sélectivité définie comme

$$
\mathrm{d}\left(\frac{E_{\mathrm{r}}}{E_{\mathrm{i}}}\right) / \mathrm{d}\left(M_{2}\right)=f\left(M_{2}\right)
$$

avec

$$
E_{\mathrm{r}}=\left[\frac{M_{1} \cos \theta+\sqrt{M_{2}^{2}-M_{1}^{2} \sin ^{2} \theta}}{M_{1}+M_{2}}\right]^{2} E_{\mathrm{i}}
$$

ou

$$
E_{\mathbf{r}}=k E_{\mathbf{i}}
$$


(r et i se référant à l'énergie rétrodiffusée et incidente, 1 et 2 au noyau incident et cible) caractérise l'aptitude pour un ion donné à assurer une meilleure ou moins bonne séparation énergétique pour deux masses adjacentes.

On a ainsi représenté sur la figure 1 la variation de ce paramètre en nous restreignant, hormis les ions ${ }^{6} \mathrm{Li}$ et ${ }^{7} \mathrm{Li}$ aux particules $\alpha$ et aux ions ${ }^{12} \mathrm{C}$ (pour $\theta=160^{\circ}$. On observe ainsi, pour $18<M_{2}<34$

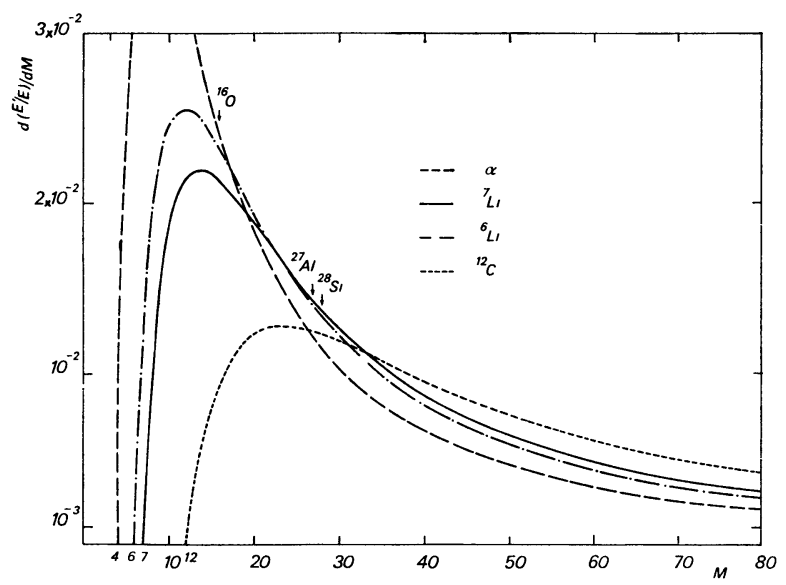

FIG. 1. - Variation de la sélectivité $\mathrm{d}\left(E^{\prime} / E\right) / \mathrm{d} M$ en fonction de la masse de l'élément analysé pour les particules $\alpha,{ }^{6} \mathrm{Li},{ }^{7} \mathrm{Li}$ et ${ }^{12} \mathrm{C}$. Certains éléments intéressant plus particulièrement les applications traitées sont repérés.

qu'il est avantageux d'utiliser des ions lithium (6 ou 7 suivant la masse). Le carbone devient plus sélectif pour $M_{2}>34$, les particules $\alpha$ assurant, de loin la meilleure sélectivité pour les éléments légers $\left(M_{2}<18\right)$. Notons que c'est dans un domaine de masses élevées, où, précisément la sélectivité est déjà très diminuée quel que soit le type de particule utilisé, que les ions carbone deviennent plus avantageux que les lithium. Cependant l'aptitude à séparer deux masses voisines apparaît déjà comme limitée par la résolution du détecteur, susceptible d'évoluer avec l'énergie nominale du faisceau. De ce point de vue, on verra qu'à plusieurs titres les particules alpha conservent un grand intérêt.

La sensibilité de la méthode liée au taux de production d'information (ou vitesse de comptage pour un type d'ion déterminé) dépend en premier lieu de la section efficace de rétrodiffusion. A cet égard, dans les domaines d'énergie accessibles aux petits accélérateurs du type Van de Graaff (de 0 à $5 \mathrm{MeV}$ ) la relation de Rutherford

$$
\begin{array}{r}
\sigma_{\text {ruth }}=\left[\frac{z_{1} z_{2} e^{2}}{2 E_{\mathrm{i}} \sin ^{2} \theta}\right]^{2} \times \\
\times \frac{\left[\cos \theta+\left[1-\left(M_{1} / M_{2} \sin \theta\right)^{2}\right]^{1 / 2}\right]^{2}}{\left[1-\left(M_{1} / M_{2} \sin \theta\right)^{2}\right]^{1 / 2}}
\end{array}
$$

apparaît comme très bien vérifiée pour les ions lithium, carbone et de masse plus élevée. Il faut cependant tenir compte du fait que l'augmentation de ce paramètre, quand l'énergie décroît, est responsable d'un rendement très important en cible épaisse avec les risques d'empilement que cela implique. On limite ces derniers par l'emploi d'ions de plus en plus lourds. C'est là un des avantages prépondérants de leur utilisation [3]. On peut aller dans certains cas jusqu'à l'élimination complète de la contribution de la matrice ou du substrat $\left({ }^{12} \mathrm{C}\right.$ avec support carbone, par exemple). Cet aspect est alors très important dans l'analyse d'éléments à l'état de traces mais n'a pas particulièrement été développé au cours de ce travail. Une électronique rapide $(0,5 \mu \mathrm{s}$ de constante de temps) mais conventionnelle a été utilisée mais à des taux de comptage relativement modérés, pour garder l'avantage d'une bonne résolution. Cet aspect demeure particulièrement intéressant pour l'étude de couches minces déposées sur des substrats très légers comme le carbone, ou autoportantes. Pour les détecteurs (barrière de surface) des résolutions de l'ordre de $15 \mathrm{keV}$, pratiquement indépendantes de l'énergie sont couramment obtenues en particules $\alpha$. De telles performances ne peuvent être obtenues pour les ions plus lourds. Plusieurs auteurs ont noté l'évolution de cette résolution avec l'énergie pour différents types d'ions : Abel et al. [3] pour ${ }^{12} \mathrm{C}$, Petterson et al. [4] pour ${ }^{16} \mathrm{O}$, Bergström et al. [5] pour ${ }^{12} \mathrm{C},{ }^{20} \mathrm{Ne},{ }^{40} \mathrm{Ar}$ et ${ }^{84} \mathrm{Kr}$. Sans avoir d'explication définitive, ce phénomène semble bien provenir d'une collection incomplète des charges dans le détecteur et paraît également lié à la variation du pouvoir d'arrêt du matériau détecteur. Cette évolution systématique de la résolution avec l'énergie des ions détectés s'accompagne d'une dégradation irréversible, fonction de la dose reçue par la jonction, probablement corrélée à la création de défauts dans le cristal détecteur. L'emploi d'ions lithium apparaît intéressant, de ce double point de vue puisque pour la gamme d'énergie des particules détectées $(250 \mathrm{keV}-2,3 \mathrm{MeV})$ cette résolution n'excède pas $30 \mathrm{keV}$ suivant la courbe expérimentale représentée (Fig. 2). Les points expérimentaux ont été obtenus à

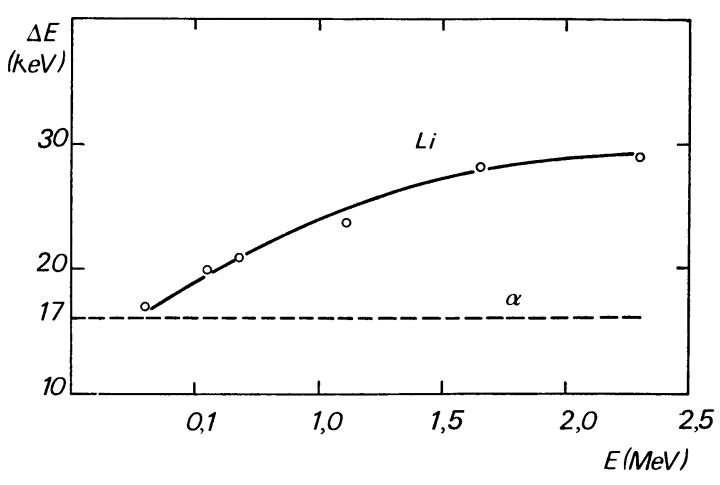

Fig. 2. - Evolution de la résolution du détecteur en fonction de l'énergie de l'ion lithium détecté.

partir de couches minces comme SiO déposé sur carbone vitreux (60 $\AA$ ) (Fig. 3) et Au également déposé sur carbone $(\sim 100 \AA)$. Une mesure directe de la réso- 


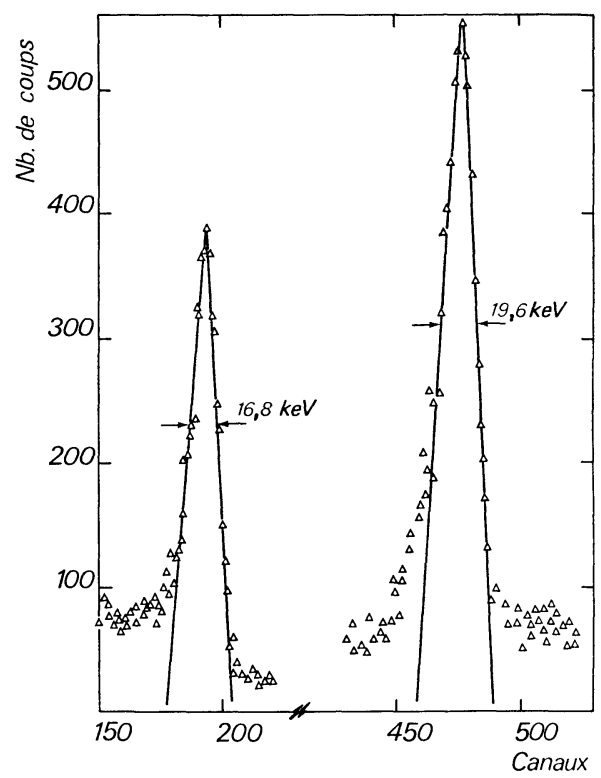

Fig. 3. - Spectre de rétrodiffusion $7 \mathrm{Li}$ à $1,9 \mathrm{MeV}, \theta=160^{\circ}$ sur une cible $\mathrm{SiO} \sim 60 \AA$ déposée sur un support de carbone et permettant une détermination de la résolution du détecteur.

lution sur faisceau [5], bien que plus délicate, peut conduire à de meilleurs résultats, compte tenu de la difficulté à réaliser des cibles suffisamment minces et des risques de diffusion dans le substrat. Ces performances expérimentales, prévisibles d'après les données de Bergström et al. [5], n'apparaissent d'autre part que peu affectées par la dose reçue puisque la résolution optimale $(17 \mathrm{keV}$ à $310 \mathrm{keV})$ est conservée après plusieurs jours de fonctionnement ininterrompu.

La résolution en profondeur de la méthode se déduit directement de la résolution expérimentale à partir du paramètre de rétrodiffusion élastique $[S]$ qui s'exprime par

$$
[S]=k\left|\frac{\mathrm{d} E}{\mathrm{~d} x}\right|_{E_{\mathrm{i}}}+\frac{1}{|\cos \theta|}\left|\frac{\mathrm{d} E}{\mathrm{~d} x}\right|_{E_{\mathrm{r}}}
$$

à partir des pouvoirs d'arrêt aux énergies incidente $E_{\mathbf{i}}$ et rétrodiffusée $E_{\mathrm{r}}$. On en déduit les valeurs des courbes $a), b$ ), c) de la figure 4 pour les éléments $\mathrm{O}, \mathrm{Al}$ et Au représentatifs des applications dont nous parlons ici. On notera en particulier que, compte tenu de la variation du pouvoir d'arrêt en fonction de l'énergie, les ions ${ }^{6} \mathrm{Li}$ conduisent aux meilleures performances, la résolution en profondeur pour les particules $\alpha$ restant meilleure que pour les ions carbone jusqu'à $2,5 \mathrm{MeV}$. Un autre avantage des ions lithium réside dans leur pouvoir d'arrêt. Ce dernier, dans le domaine énergétique accessible à nos machines, passe par un maximum à partir duquel il décroît lentement, pour la plupart des éléments (particulièrement pour les lourds). On pourra donc, comme l'illustre la figure 4, maintenir une résolution en profondeur optimale et accroître la sélectivité en travaillant à l'énergie la plus haute possible.

3. Applications. - 3.1 DisPoSITIFS EXPÉRIMENTAUX. - Des sources solides de lithium 7 ou 6 (enrichisse-

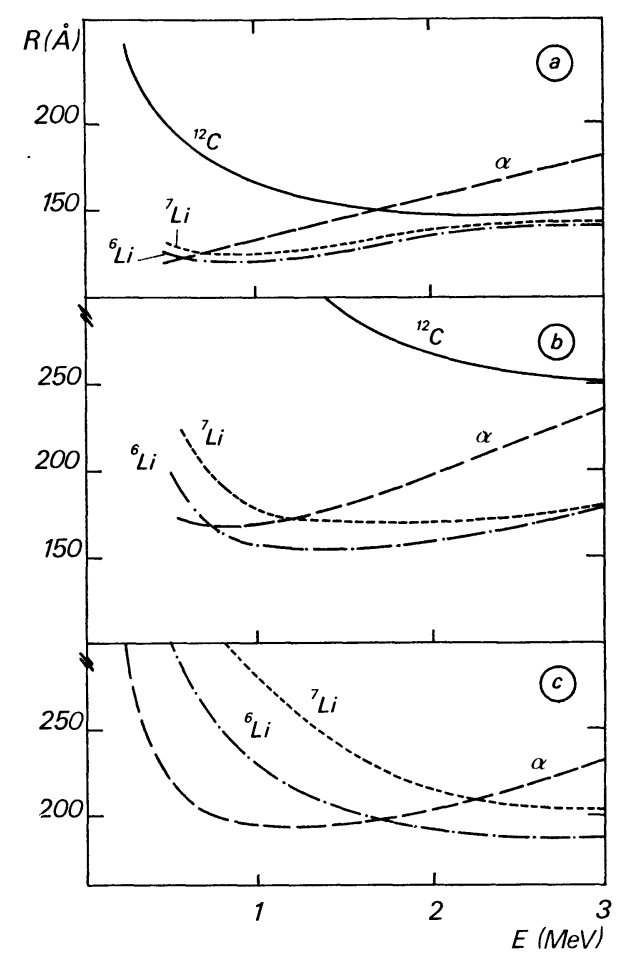

Fig. 4. - Evolution de la résolution en profondeur en fonction de l'énergie : a) Au dans $\mathrm{Au} ; b$ ) $\mathrm{Al}$ dans $\mathrm{Al}_{2} \mathrm{O}_{3} ; c$ ) $\mathrm{O}$ dans $\mathrm{Al}_{2} \mathrm{O}_{3}$. Les résolutions des détecteurs sont prises à $17 \mathrm{keV}$ pour les $\alpha$, suivant la courbe de la figure 2 pour $\mathrm{Li}$, d'après [5] pour ${ }^{12} \mathrm{C}$.

ment à $99 \%$ ) équipent l'accélérateur Van de Graaff de l'Institut de Physique Nucléaire de Lyon, permettant pour ces ions une énergie maximale de $2 \mathrm{MeV}$. L'extension du domaine d'énergie sur le Van de Graff $4 \mathrm{MeV}$ de l'I. P. N. est limitée par les performances de l'aimant d'analyse à $90^{\circ}$ qui ne permet d'accéder respectivement qu'à $2,4 \mathrm{MeV}{ }^{7} \mathrm{Li}$ ou $2,6 \mathrm{MeV}{ }^{6} \mathrm{Li}$. Par contre cette machine dévie des particules $\alpha$ jusqu'à l'énergie maximale de $4 \mathrm{MeV}$. Les détecteurs utilisés pour ces études sont du type barrière de surface, $25 \mathrm{~mm}^{2}$, $100 \mu \mathrm{m}, 100 \mathrm{nA}$ de courant de fuite à $60 \mathrm{~V}$ de polarisation et ont une résolution $\alpha$ de $14 \mathrm{keV}$ (source d'américium). L'électronique associée est conventionnelle (Tennelec : préampli TC 163 ampli TC 205), la constante de temps étant fixée à $0,5 \mu \mathrm{s}$. Le traitement des spectres est effectué, après analyse dans un convertisseur HP, sur un calculateur HP 2116. Un tel ensemble de détection a déjà été décrit par ailleurs [7].

\subsection{ETUDE DES COUCHES D'OXYDATION ANODIQUE} DE L'Aluminium. - Dans la technologie de fabrication des condensateurs électrolytiques, la fabrication du diélectrique par une technique d'oxydation anodique dite de formation, conduit à l'élaboration de couches barrière constituant l'anode du condensateur. La qualité de ce dernier va dépendre de cette couche barrière classiquement obtenue à l'issue d'un processus en trois étapes: A) Formation sous tension ; B) Imprégnation de la feuille formée, ou passivation sans tension dans un bain phosphorique $\mathrm{H}_{3} \mathrm{PO}_{4}$ (incor- 
poration d'ions phosphore); C) Post-formation (restructuration de la couche). Les bains utilisés pour les stades $\mathrm{A}$ et $\mathrm{C}$ sont essentiellement phosphorique et carboxylique pour des tensions inférieures à $300 \mathrm{~V}$.

L'analyse par rétrodiffusion élastique permet d'obtenir, à chacune de ces étapes, les informations fondamentales que constituent i) la stœchiométrie et l'épaisseur de la couche ou la variation de composition de la phase oxydée, ii) la répartition de l'impureté majeure du bain de formation. La figure 5 illustre cette possi-

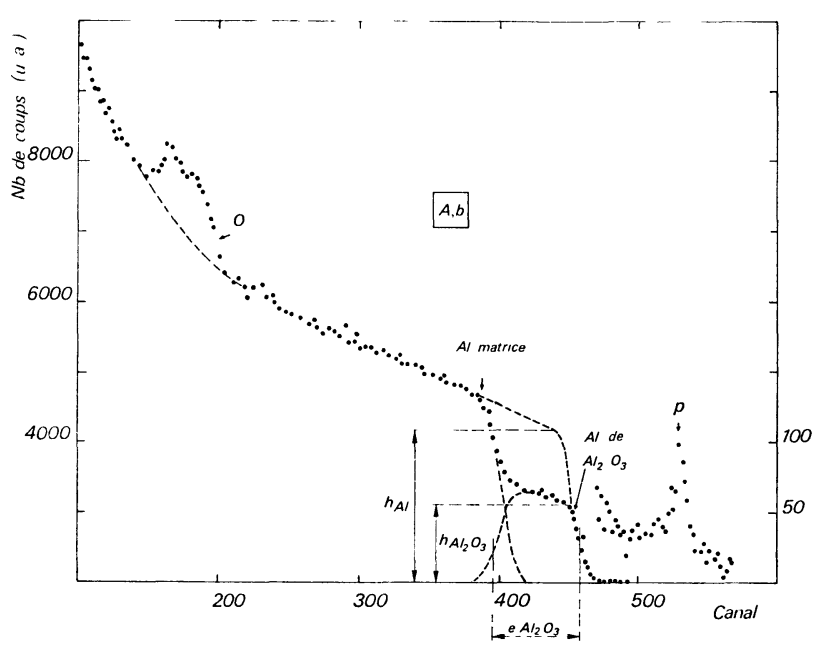

FIG. 5. - Spectre de rétrodiffusion élastique à $160^{\circ}$ d'ions $7 \mathrm{Li}$ sur une couche formée à $60 \mathrm{~V}$ en carboxylique $(\mathrm{A}(\mathrm{b}))$. On a différencié la contribution de l'oxygène de l'aluminium matrice et de l'aluminium de l'oxyde. On a également superposé le spectre d'aluminium permettant le calcul des hauteurs de plateaux.

bilité de détermination simultanée de ces paramètres fondamentaux avec le spectre de rétrodiffusion élastique à $160^{\circ}$ d'ions ${ }^{7} \mathrm{Li}$ de $1,9 \mathrm{MeV}$ sur une couche formée à $60 \mathrm{~V}$ en milieu carboxylique. L'oxydation de la couche est traduite par la marche sur le front de montée de l'aluminium et la superposition sur le spectre carac téristique de la matrice d'aluminium du pavé correspondant à la rétrodiffusion sur l'oxygène. De même une contamination initiale en phosphore est traduite par un pic fin à plus haute énergie. La déconvolution schématique représentée sur la figure 5 illustre la possibilité de mesure de l'épaisseur de cette couche d'oxyde, la meilleure précision étant évidemment obtenue à partir du pavé d'aluminium dans $\mathrm{Al}_{2} \mathrm{O}_{3}$. La largeur énergétique du palier conduit à la détermination de l'épaisseur par l'intermédiaire des relations énergie-profondeur déduites des pouvoirs d'arrêt de $\mathrm{Li}$ dans $\mathrm{Al}_{2} \mathrm{O}_{3}$ déterminés à partir de la loi pondérale de Bragg et des tables de Northcliffe et Schilling [6]. Pour des interfaces nettes, la précision s'étage de 10 à $3 \%$ pour des épaisseurs comprises entre 400 et $1000 \AA ̊$. Au-delà de ces valeurs, l'insuffisance de la largeur du palier et l'effet de straggling constituent une limitation. Dans un cas exposé ci-après, l'interface métal-oxyde est très perturbée. Les variations de densité du milieu n'autorisent alors qu'à donner un ordre de grandeur.

La stœchiométrie de la couche peut être déterminée par simple comparaison de la hauteur des paliers ainsi que l'indique également la figure 5. Pour une stœchiométrie $\mathrm{Al}_{2} \mathrm{O}_{3}$ le rapport de ces hauteurs s'exprime par :

$$
\frac{h_{\mathrm{Al}}}{h_{\mathrm{Al}_{2} \mathrm{O}_{3}}}=\frac{n_{\mathrm{atAl} / \mathrm{g} \text { dans Al }}}{n_{\mathrm{atAl} / \mathrm{g} \text { dans } \mathrm{Al}_{2} \mathrm{O}_{3}}} x \frac{(\Delta E / \Delta x)_{\mathrm{Al}_{2} \mathrm{O}_{3}}}{(\Delta E / \Delta x)_{\mathrm{Al}}}
$$

à l'énergie maximale de rétrodiffusion, $\Delta E$ correspondant au contenu d'un canal soit à une épaisseur $\Delta x$. Avec

$$
\frac{\Delta E}{\Delta x} \simeq[S]
$$

défini au chapitre précédent, on aura

$$
\frac{h_{\mathrm{Al}}}{h_{\mathrm{Al}_{2} \mathrm{O}_{3}}}=1,973
$$

pour ${ }^{7} \mathrm{Li}$ de $1,9 \mathrm{MeV}$ et $\theta=160^{\circ}$.

Avec une précision de l'ordre de $6 \%$ l'écart à la stœchiométrie n'excède jamais $3 \%$ pour une formation du type A.

L'influence du traitement B apparaît alors sur les figures 6 et 7 (spectres $B$ ) avec une réduction prévisible de l'épaisseur et un écart marqué à la stœchiométrie (sous-stœchiométrie en $\mathrm{Al}$ ). Une forte concentration en phosphore apparaît dans chaque cas plus marquée et plus superficielle pour l'échantillon ayant subi une formation phosphorique. Pour le traitement C (Fig. 6 et 7 , spectres $\mathrm{C}$ ), on note pour le traitement phosphorique une restauration de la couche en stœchiométrie et en épaisseur accompagnée d'une diminution importante de la concentration en phosphore par ailleurs plus uniforme. Par contre, le traitement carboxylique détériore complètement la couche : l'interface est détruite et la zone perturbée est de l'ordre de $3000 \AA$. Cette constatation peut également être faite à partir de la

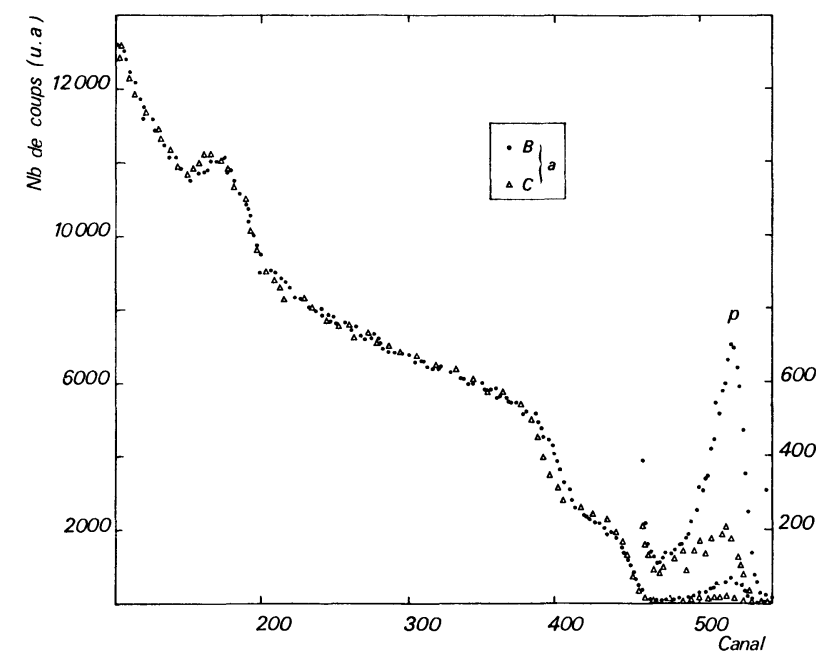

FIG. 6. - Spectres de rétrodiffusion élastique à $160^{\circ}$ d'ions $7 \mathrm{Li}$ sur les échantillons correspondant aux traitements $B(a)$ et $C(a)$ en phosphorique. 


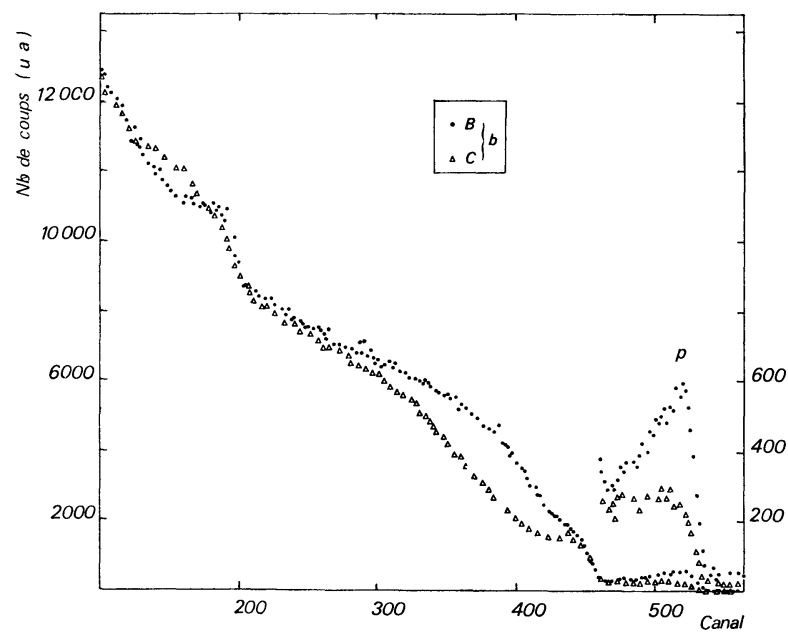

Fig. 7. - Spectres de rétrodiffusion élastique à $160^{\circ}$ d'ions $7 \mathrm{Li}$ sur les échantillons correspondant aux traitements $\mathrm{B}(\mathrm{b})$ et $\mathrm{C}(\mathrm{b})$ en carboxylique.

distribution de l'oxygène. L'ensemble de ces résultats est consigné dans le tableau I.

A $1,9 \mathrm{MeV}$ l'emploi des ions ${ }^{7} \mathrm{Li}$ ne permet l'analyse du phosphore que sur une épaisseur de l'ordre de $700 \AA$, inférieure à celle de la couche d'oxyde, mais avec une résolution en profondeur de $150 \AA$. Cette zone peut être étendue à $1700 \AA$ avec des particules $\alpha$ de $3,5 \mathrm{MeV}$ au détriment de la résolution en profondeur alors égale à $350 \AA$. Cette complémentarité d'emploi des deux types de particules est illustrée par la figure 8 pour le traitement $\mathrm{C}$ en carboxylique.

Ces résultats ont été confrontés à ceux d'autres méthodes physiques d'analyse dont l'emploi de la réaction ${ }^{27} \mathrm{Al}(\mathrm{p}, \gamma)$. La résonance très fine $(\Gamma<100 \mathrm{eV})$ de cette réaction à $992 \mathrm{keV}$ a déjà été utilisée par Amsel

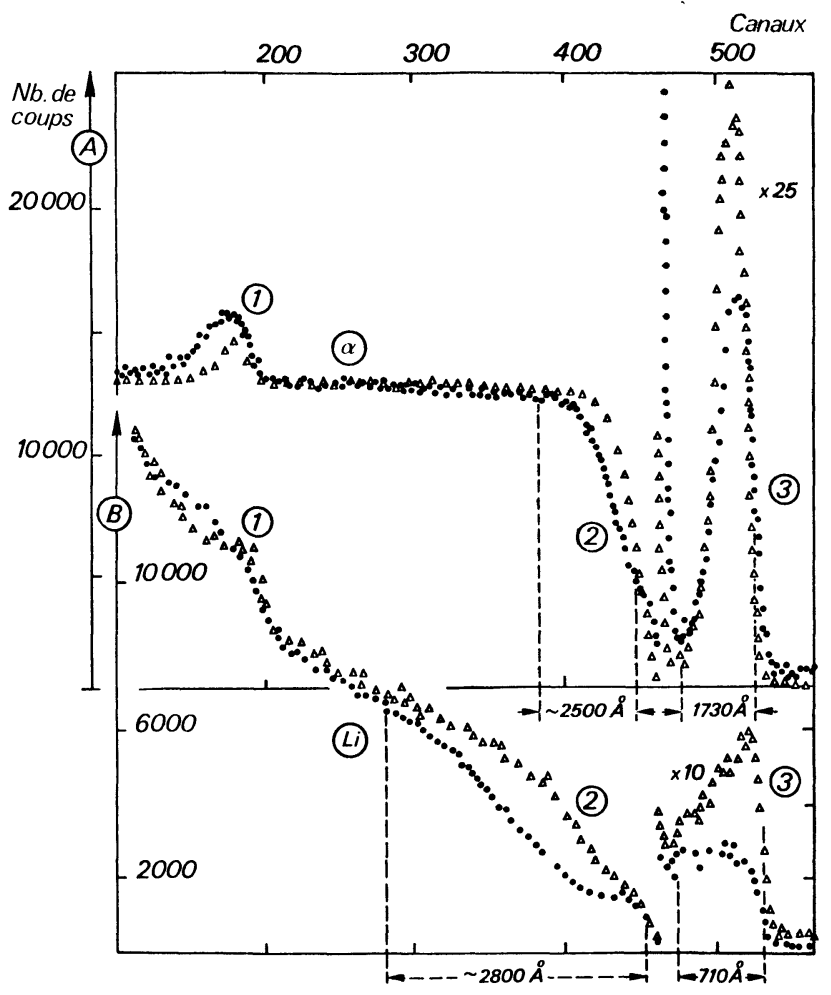

FIg. 8. - Comparaison des spectres obtenus sur les échantillons aux stades $B(\Delta \Delta \Delta)$ et $C\left(\bullet^{\circ}\right)$ du traitement carboxylique en rétrodiffusion élastique de particules $\alpha$ de $3,5 \mathrm{MeV}(\alpha)$ et d'ions lithium de 1,9 MeV (Li). On peut voir les variations identiques de la répartition en oxygène 1 , en aluminium 2 et en phosphore 3.

et al. [8] pour déterminer la stoechiométrie de couches d'oxydation anodique d'aluminium. Avec une largeur expérimentale de $1 \mathrm{keV}$ pour cette résonance, la résolution en profondeur est de $135 \AA$ en surface mais se

\section{TABleAU I}

\begin{tabular}{|c|c|c|c|c|c|c|c|}
\hline & \multicolumn{2}{|c|}{$\begin{array}{l}\text { Ecart à la stœchiométrie } \\
\mathrm{Al}_{2} \mathrm{O}_{3}\end{array}$} & \multicolumn{5}{|c|}{$\begin{array}{r}\text { Epaisseurs concernées (théorique } 822 \AA / 60 \mathrm{~V} \text { ) } \\
\text { Mesures de capacités }\end{array}$} \\
\hline - & R. E. ${ }^{7} \mathrm{Li}$ & ${ }^{27} \mathrm{Al}(\mathrm{p}, \gamma)$ & R. E. ${ }^{7} \mathrm{Li}$ & ${ }^{27} \mathrm{Al}(\mathrm{p}, \gamma)$ & Ellipsométrie & Electrolyte & solide \\
\hline$A(b)$ & $\sim 1,8 \%$ & $\sim 3 \%$ & $820 \pm 25 \AA$ & $812 \pm 70 \AA$ & $\sim 1000 \AA$ & $\begin{array}{c}650 \AA \\
\left(11 \mu \mathrm{F} / \mathrm{dm}^{2}\right)\end{array}$ & \\
\hline $\mathrm{B}(\mathrm{b})$ & $\sim 23,8 \%$ & & $530 \pm 50 \AA$ & $540 \pm 100 \AA$ & $\sim 650 \AA$ & & \\
\hline$C(b)$ & $\sim 37 \%$ & $\sim 32 \%$ & $\begin{array}{l}\text { entre } 1000 \\
\text { et } 3000 \AA\end{array}$ & $>1000 \AA$ & $\sim 1800 \AA$ & $\begin{array}{c}650 \AA \\
\left(11 \mu \mathrm{F} / \mathrm{dm}^{2}\right)\end{array}$ & $\begin{array}{c}1100 \AA \\
\left(6,5 \mu \mathrm{F} / \mathrm{dm}^{2}\right)\end{array}$ \\
\hline $\mathbf{B}(\mathrm{a})$ & $\sim 10 \%$ & & $610 \pm 50 \AA$ & & $\sim 800 \AA$ & $\begin{array}{c}500 \AA \\
\left(14 \mu \mathrm{F} / \mathrm{dm}^{2}\right)\end{array}$ & \\
\hline$C(a)$ & $\sim 1 \%$ & & $830 \pm 25 \AA$ & & $\sim 950 \AA$ & $\begin{array}{c}700 \AA \\
\left(10,1 \mu \mathrm{F} / \mathrm{dm}^{2}\right)\end{array}$ & $\begin{array}{c}750 \AA \\
\left(9,4 \mu \mathrm{F} / \mathrm{dm}^{2}\right)\end{array}$ \\
\hline
\end{tabular}

$\mathrm{A}(\mathrm{b})$ Témoin formé à $60 \mathrm{~V}$ en électrolyte type carboxylique $\mathrm{R}-\mathrm{C}_{\mathrm{OH}}$

$\mathrm{B}$ (b) Incorporation de $\mathrm{P}$ dans la couche par voie chimique à partir de la formation en carboxylique.

$\mathrm{C}$ (b) Reformation à $60 \mathrm{~V}$ de $\mathrm{B}(\mathrm{b})$ en carboxylique.

$\mathrm{B}(\mathrm{a})$ Incorporation de $\mathrm{P}$ à partir de la formation en électrolyte type phosphorique/phosphate.

$\mathrm{C}(\mathrm{a})$ Reformation à $60 \mathrm{~V}$ de $\mathrm{B}(\mathrm{a})$ en phosphorique.

REVUE DE PHYSIQUE APPLIQUÉE. - T. 11, No 1, JANVIER 1976 
détériore rapidement avec le straggling. De la même façon la stœchiométrie se déduit du rapport des hauteurs de plateaux du rendement de la réaction quand on analyse par déplacement de la résonance (variation de l'énergie nominale), l'aluminium de la couche oxydée, puis l'aluminium de la matrice. De même, la zone énergétique pour laquelle le rendement de la réaction est constant pour $\mathrm{Al}$ dans $\mathrm{Al}_{2} \mathrm{O}_{3}$ permet de mesurer l'épaisseur de la couche. Cependant, avec l'épaisseur de la couche, l'intervention du straggling et l'apparition de nouvelles résonances à plus haute énergie font décroître la précision. L'accord tant pour la stœchiométrie que pour l'épaisseur demeure cependant excellent pour les couches à interface définie, comme le montre le tableau I. Il faut noter que la mise en œuvre de cette dernière méthode nécessite un temps environ dix fois supérieur à la précédente. Enfin deux méthodes spécifiques pour l'alumine permettent également d'accéder aux épaisseurs : l'ellipsométrie qui suppose la couche homogène et d'indice de réfraction connu et les mesures de capacité en contre-électrode liquide ou solide. On constate (Tableau I) que ces résultats sont pour la plupart cohérents avec ceux obtenus par rétrodiffusion élastique et réaction ${ }^{27} \mathrm{Al}(\mathrm{p}, \gamma)$ encore que systématiquement supérieurs (ellipsométrie) ou inférieurs (capacités). Le plus marquant reste cependant le désaccord sur l'épaisseur déterminée par mesure capacitive en contre-électrode liquide, pour la couche ayant subi le traitement $\mathbf{C}$ en carboxylique qui manifeste par ailleurs un comportement électrique satisfaisant [9]. Il est incontestable que dans ce cas typique, la rétrodiffusion élastique seule lève cette incertitude sur les mesures électriques et complète les résultats, sujets à caution, de l'ellipsométrie.

3.3 ETUDE DE COUCHES MINCES. - On retrouve pour l'étude des couches minces les mêmes avantages à utiliser la rétrodiffusion élastique des ions lithium. La meilleure résolution en profondeur permet la mesure d'épaisseurs de couches très minces ( $<500 \AA)$ soit par l'élargissement du pic caractéristique d'un des éléments du composé soit par le recul du front du substrat sur lequel est déposée la couche. La précision de cette détermination est meilleure que dans le cas précédent (couches épaisses), puisqu'obtenue à partir d'un pavé massif et non d'une marche. La principale indétermination réside, là encore, dans la connaissance des pouvoirs d'arrêt des particules pour des milieux plus ou moins complexes. La loi de Bragg demande en particulier dans le cas de certains composés, à être vérifiée expérimentalement. Ceci est d'autant plus vrai pour les oxydes ou nitrures présentés ici avec l'utilisation d'un pouvoir d'arrêt déterminé pour un élément à l'état gazeux [10]. L'épaisseur maximale analysable dépend évidemment des constituants de la couche, mais on a vu qu'en se plaçant à la plus haute énergie possible, on étendait ce domaine tout en gardant la résolution en profondeur optimale. Ces considérations sont illustrées par la figure 9 où sont donnés les spectres obtenus à

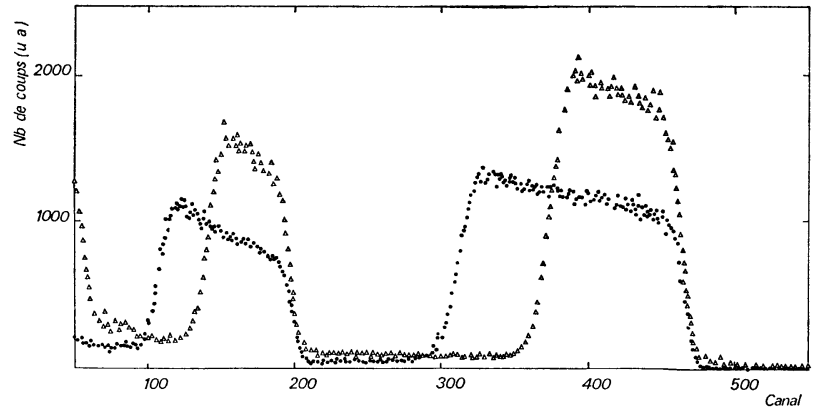

Fig. 9. - Spectre caractéristique de la rétrodiffusion d'ions ${ }^{7} \mathrm{Li}$ à $E=1,9 \mathrm{MeV}, \theta=160^{\circ}$. En points noirs : couche de $\mathrm{Al}_{2} \mathrm{O}_{3}$ $2000 \AA$ autoportante. En triangles : couche évaporée de $1200 \AA$, sur support de carbone.

$1,9 \mathrm{MeV}{ }^{7} \mathrm{Li}$ sur des couches de 1200 et $2000 \AA$ de $\mathrm{Al}_{2} \mathrm{O}_{3}$ respectivement autoportante et déposée sur un support de carbone. Pour les couches $\mathrm{SiO}, \mathrm{SiO}_{2}$ et $\mathrm{Al}_{2} \mathrm{O}_{3}$, cette épaisseur est de l'ordre de 3500 à $4000 \AA$ si l'on étudie la contribution de l'aluminium ou du silicium, mais peut être plus faible dans le cas où diverses impuretés majeures sont présentes. Par exemple pour le nitrure d'aluminium obtenu par pulvérisation cathodique diode en milieu argon [11] la séparation aluminium-argon nécessite $2300 \AA$ au plus et la séparation azote-oxygène $1250 \AA$.

Le plus important dans cette analyse de couches minces reste cependant la mesure de stœchiométrie avec une grande précision. Etant donné la validité de la relation de Rutherford à moins de $1 \%$, cette détermination à partir de l'intégrale des pics des éléments de la couche aura une précision essentiellement dépendante de la statistique du comptage. On a ainsi pu confirmer les résultats obtenus en $(\mathrm{d}, \mathrm{p})$ sur $\mathrm{SiO}$ (écart à la stœchiométrie théorique $<2 \%$ ) et sur $\mathrm{Al}_{2} \mathrm{O}_{3}$ ( $<3 \%$ ) [12], [13] mais en se dispensant d'étalons, ce qui constitue l'avantage prépondérant de la méthode. Pour les mesures absolues du nombre d'atomes $/ \mathrm{cm}^{2}$ on peut toujours se ramener à un étalon simple d'épaisseur massique parfaitement déterminée par toute autre méthode et utiliser là aussi la relation de Rutherford. La détermination de profils de répartition d'un élément du composé ou d'une impureté de ces couches minces est également affinée par l'emploi d'ions lithium, et particulièrement pour les éléments légers, comme on l'a vu au niveau de la résolution en profondeur. On peut ainsi accéder à l'interprétation de certaines croissances de couches comme dans l'exemple de la figure 10, avec un spectre de rétrodiffusion d'ions ${ }^{6} \mathrm{Li}$ de $1,9 \mathrm{MeV}$ obtenu sur une cible de nitrure d'aluminium [11]. Cette dernière a été obtenue par pulvérisation réactive diode, sans pré-pulvérisation de la cathode avant dépôt, et sur un substrat refroidi par une circulation d'eau. La contamination de la cathode est responsable d'un dépôt d'alumine en début de pulvérisation, le nitrure étant synthétisé dès que la cathode est propre. L'importante quantité d'oxygène demeurant dans la couche de nitrure est ici probablement due à une oxydation de 


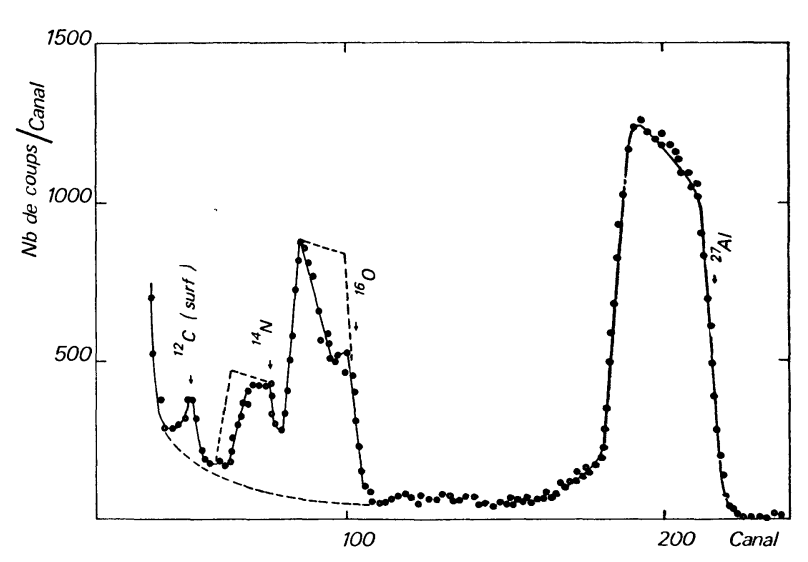

FIG. 10. - Spectre de rétrodiffusion élastique ${ }^{6} \mathrm{Li}$ sur une couche en nitrure d'aluminium, montrant l'évolution de la couche d'oxyde pendant la pulvérisation.

l'excès d'aluminium obtenu lors des dépôts sur un substrat refroidi.

Le dernier volet de cette revue d'applications de la rétrodiffusion élastique d'ions lithium concerne la détermination de la profondeur d'implantation d'ions dans les couches minces, et plus particulièrement ici d'ions ${ }^{16} \mathrm{O}^{+}$d'énergie comprise entre 10 et $40 \mathrm{keV}$, dans $3000 \AA$ de monoxyde de silicium SiO [14]. La figure 11 montre les spectres représentatifs de la zone non implantée et implantée $\left(40 \mathrm{keV}{ }^{16} \mathrm{O}^{+}\right.$dose de $\left.6 \mu \mathrm{g} / \mathrm{cm}^{2}\right)$. On peut noter pour la zone implantée que l'excès d'oxygène correspond à un défaut de silicium.

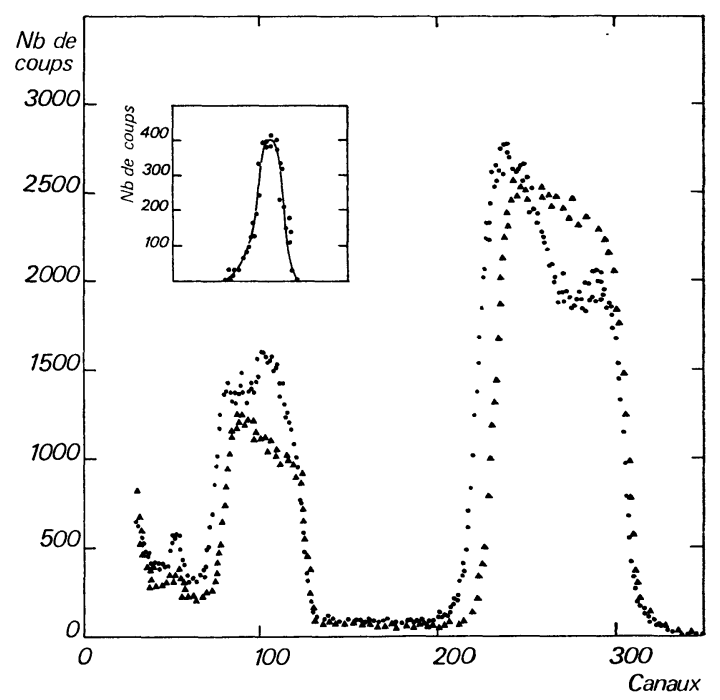

Fig. 11. - Spectre de rétrodiffusion élastique d'ions $7 \mathbf{L i}$ sur une couche de SiO $300 \AA$ non implantée $(\boldsymbol{\Delta} \mathbf{\Delta})$ et implantée en $16 \mathrm{O}^{+} 40 \mathrm{keV}(\ldots)$. Les spectres superposés ne sont pas normalisés pour permettre une meilleure différenciation. En encadré, on a donné la répartition de l'oxygène implanté par soustraction des pics correspondant à l'oxygène.
La modification de la composition du film inhérente à l'implantation traduit l'élargissement des pics représentés par ailleurs pour deux intégrations du courant $\mathrm{Li}$ total légèrement différentes. Après normalisation des spectres et soustraction de la contribution en oxygène, on donne (cf. encadré de la Fig. 11) la courbe de répartition en profondeur des ions oxygène implantés. La surface intégrée est proportionnelle à la dose implantée et la profondeur de pénétration peut être mesurée. Un écart maximal de $20 \%$ pour des ions de $10 \mathrm{keV}$ à $40 \mathrm{keV}$ avec la théorie de Lindhart pour des composés à deux corps apparaît satisfaisant étant donné que ni la modification continue de stœchiométrie ni l'autodiffusion possible des ions dans la couche ne sont pris en compte. L'intégration des pics de silicium des couches implantée et non implantée permet par ailleurs de vérifier qu'il n'y a pas de sputtering aux énergies d'implantation utilisées. Enfin la stœchiométrie de la zone implantée a pu être mesurée comme $N_{\mathrm{O}} / N_{\mathrm{Si}}=1,31$ à $40 \mathrm{keV}$ pour une valeur théorique de 1,30.

4. Conclusion. - L'emploi d'ions lithium en rétrodiffusion élastique semble constituer un apport intéressant à une technique actuellement très développée pour l'analyse des couches minces et des surfaces. Compte tenu des limitations inhérentes aux ions de plus en plus lourds au niveau de la résolution des détecteurs, ces ions lourds légers semblent actuellement réaliser le meilleur compromis entre sélectivité, sensibilité, résolution en profondeur et profondeur d'analyse. Comme pour la majorité des ions lourds, une étude expérimentale plus fine reste nécessaire pour déterminer avec une meilleure précision les pouvoirs d'arrêt et parcours, surtout dans les milieux complexes. A l'exception des mesures de stœchiométrie globale, la qualité de toutes les informations en dépend. De même, l'évolution de la résolution des détecteurs à barrière de surface avec l'énergie de l'ion détecté doit être établie avec une plus grande précision. Ces deux études font l'objet de travaux en cours. Il est toutefois intéressant de souligner à nouveau que pour ces ions lithium, le domaine d'énergie de l'ordre du $\mathrm{MeV}$, c'est-à-dire parmi les plus accessibles aux petits accélérateurs, conduit au meilleur compromis sur l'ensemble des performances attendues. De ce point de vue, l'extension du domaine d'énergie de l'accélérateur Van de Graaff de l'I. P. N. Lyon de 2,6 MeV actuellement (limitation au niveau de l'aimant à $\left.90^{\circ}\right)$ à $4 \mathrm{MeV}$ constituerait un gain appréciable. Sans préjuger des performances optimales des détecteurs actuels, il apparaîtrait également intéressant d'entreprendre une investigation des ions de masse voisine, comme ${ }^{9} \mathrm{Be}$ et surtout ${ }^{10} \mathrm{~B}$ ou ${ }^{11} \mathrm{~B}$.

\section{Bibliographie}

[1] Luthereau, F., Tousset, J., Bull. Soc. Chim. de France $\mathrm{n}^{\circ} 11$ (1968) 4724.

[2] MAYer, J. W., Ziegler, J. F., ed. Ion-Beam surface layer analysis Intern. Conf., June 1973 (Elsevier Publ., Amsterdam) 1974.
[3] Abel, F., Amsel, G., Bruneaux, M., Cohen, C., MauRel, A., Rigo, S., Roussel, J., J. Rad. Chem. 16 (1973) 587.

[4] Petterson, S., Tove, P. A., Meyer, O., Sundevist, B., Johansson, A., Thin Solid Films 19 (1973) 157 
[5] Bergström, I., Buorkqvist, K., DomeiJ, B., Fladda, G., Can. J. Phys. 46 (1968) 2679.

[6] NorthCliffe, L. C., Schilling, R. F., Nucl. Data Tables 7 (1970) 233.

[7] Thomas, J. P., Thèse Doct. ès-Sci. Phys., Lyon (1974).

[8] Amsel, G., Nadai, J. P., D’Artemare, E., David, D., Girard, E., Moulin, J., Nucl. Instrum. Methods 92 (1971) 481.

[9] Thomas, J. P., Fallavier, M., Cachard, A., Badia, M., Rapport DGRST (1975) 73-7-1545.
[10] Thomas, J. P., Cachard, A., Fallavier, M., Dupin, J. P., MARSAUd, S., (à paraître).

[11] Tardy, J., Thèse Doct. Spéc. $3^{\mathrm{e}}$ Cycle, Lyon (1975).

[12] Cachard, A., Pivot, J., Roger, A., Talvat, M., ThoMAS, J. P., Revue Phys. Appl. 6 (1971) 279.

[13] Diaine, C., Roger J. A., Pivot, J., Cachard, A., DuPuY, C., Congrès AVISEM, Versailles (1971).

[14] Pivot, J., Morelli, D., Cachard, A., Tardy, J., Roger, J. A., Thomas, J. P., Soumis à «Intern. Conf. on the Applications of ion beams to materials » Warwick, Septembre 1975. 